
\title{
$\angle$ Research Square \\ Phenotypic Clusters On Computed Tomography Reflects Asthma Heterogeneity And Severity
}

\section{Sujeong Kim ( $\nabla$ crystal1837@gmail.com )}

Kyungpook National University School of Medicine https://orcid.org/0000-0002-2494-9216

\section{Sanghun Choi}

Kyungpook National University

\section{Taewoo Kim}

Kyungpook National University

\section{Kwang Nam Jin}

SNU SMG Boramae Medical Center: Seoul National University Seoul Metropolitan Government Boramae Medical Center

\section{Sang-Heon Cho}

Seoul National University College of Medicine

\section{Chang Hyun Lee}

Seoul National University College of Medicine

\section{Hye-Ryun Kang}

Seoul National University College of Medicine

\section{Keywords:}

Posted Date: July 27th, 2021

DOl: https://doi.org/10.21203/rs.3.rs-713966/v1

License: (c) (1) This work is licensed under a Creative Commons Attribution 4.0 International License. Read Full License

Version of Record: A version of this preprint was published at World Allergy Organization Journal on February 1st, 2022. See the published version at https://doi.org/10.1016/j.waojou.2022.100628. 


\section{Abstract}

The authors have requested that this preprint be removed from Research Square. 